\title{
ON CURVES KINEMATICALLY RELATED TO A GIVEN CURVE *
}

BY H. PORITSKY

In a space $S$ is given a curve $C$. With any point $P$ on it is associated a position of the moving trihedron formed by the tangent, principal normal, and binormal to the curve at $P$. We might call an indicatrix trihedron a trihedron whose axes pass through a fixed point, and are parallel to those of the moving trihedron, and denote it by $I$. As the point $P$ describes the curve, the indicatrix trihedron will rotate about (lines passing through) its vertex. This motion, or the motion of a space rigidly connected to the indicatrix trihedron, with reference to the space $S$, we shall denote by $[I, S]$. The problem solved in this paper is, to find all the curves $C^{\prime}$ such that their indicatrix trihedra $I^{\prime}$ (when drawn with the vertex as $I$ ) will experience a motion $\left[I^{\prime}, S\right]$ identical with the motion $[I, S]$; in other words, to find curves $C^{\prime}$, whose points $P^{\prime}$ can be made to correspond to $P$ so that, as the curves are described by corresponding points, their indicatrix trihedra $I, I^{\prime}$ remain relatively invariant. Certain interesting families of curves $C^{\prime}$ are shown to exist. A kinematic method of treatment has been adopted.

Denote unit vectors along the tangent, principal normal, and binormal of $C$ by $i, j, k, \uparrow$ respectively. It is known that the motion $[I, S]$ is completely characterized by the fact that there is no component of rotation along the principal normal $j . \quad$ The components of the rotation along $i, k$, if $P$ describes $C$ with unit velocity, may be identified with the torsion $\tau$ and the curvature $\kappa$. If the velocity of $P$ is not unity but $v=d s / d t$ ( $s$ is arc length, $t$ time), the components of rotations become $v \tau, v \kappa$. Thus the rotation vector always lies in the plane $\pi$, through $i, k$, and parallel to the rectifying plane. It will be noticed that $\pi$ is a plane of (i.e., fixed relative to) the moving space $I^{\prime}$.

* Presented to the Society, December 28, 1922.

$\dagger$ Clarendon type will be used to denote vectors.

† Darboux, Théorie des Surfaces, 2d ed., vol. I, p. 13. 
We must now consider two cases:

1. The motion $[I, S]$ consists of a rotation about a fixed line.

2. The motion $[I, S]$ may be generated by rolling a cone fixed in $I$ upon a cone fixed in $S$. The cones are the loci of instantaneous axes of revolution in $I$ and $S$ respectively. Case 1 is the special instance when the cones degenerate into lines.

Since, as just mentioned, the rotation vector has no component along $j$, the rolling cone, whose elements bear the rotation vectors, reduces to the plane $\pi$ (case 2 ), or else the fixed axis of rotation is a line in $\pi$ (case 1 ). In the latter case the components of rotation along $i, k$ have a fixed ratio. Hence

$$
\frac{\kappa}{\tau}=\text { const. }=\tan \alpha,
$$

where $\alpha$ is the angle which the fixed rotation axis makes with $i$. $\quad C$ is known to be a cylindrical helix. Leaving this case for later consideration, we shall first consider the general case.

Let primed letters indicate objects referring to $C^{\prime}$ of the same kind as the unprimed letters denote for $C$. Let corresponding points on the two curves be given by the same value of $t$; thus $P(t), P^{\prime}(t)$ will denote corresponding points on $C, C^{\prime}$, while $P, P^{\prime}$ denote merely any two points on $C, C^{\prime}$; similarly $\boldsymbol{i}(t), \boldsymbol{i}^{\prime}(t)$ will be used for unit tangents at two corresponding points, and so forth.

If the motions $[I, S],\left[I^{\prime}, S\right]$ be identical, the fixed and the rolling cones for the two motions, as well as the angular velocities, must be the same. But since the rolling cones reduce to the planes $\pi, \pi^{\prime}$ which are parallel to the rectifying planes at $P(t), P^{\prime}(t)$, respectively, $\pi(t), \pi^{\prime}(t)$ must coincide, and so must the principal normals $j(t), j^{\prime}(t)$ which are perpendicular to $\pi, \pi^{\prime}$. The rotation by means of which $I(t)$ is transformed into $I^{\prime}(t)\left(\boldsymbol{i}(t), \boldsymbol{j}(t), \boldsymbol{k}(t)\right.$ going into $\left.\boldsymbol{i}^{\prime}(t), \boldsymbol{j}^{\prime}(t), \boldsymbol{k}^{\prime}(t)\right)$ is therefore about the line bearing $j(t), j^{\prime}(t)$, and is of a constant angle $\theta$, since the two indicatrix trihedra are relatively fixed. Resolving the unit vectors of $I^{\prime}$ along those of $I$, we get 


$$
\begin{aligned}
\boldsymbol{i}^{\prime}(t) & =\boldsymbol{i}(t) \cos \theta+\boldsymbol{k}(t) \sin \theta, \\
\boldsymbol{j}^{\prime}(t) & =\boldsymbol{j}(t), \\
\boldsymbol{k}^{\prime}(t) & =-\boldsymbol{i}(t) \sin \theta+\boldsymbol{k}(t) \cos \theta,
\end{aligned}
$$

$\theta$ being independent of $t$.

Equating the rotation vectors

(2) $v^{\prime}(t)\left[\boldsymbol{\tau}^{\prime}(t) \boldsymbol{i}^{\prime}(t)+\kappa^{\prime}(t) \boldsymbol{k}^{\prime}(t)\right]=v(t)[\tau(t) \boldsymbol{i}(t)+\kappa(t) \boldsymbol{k}(t)]$,

we have identified the fixed cones and the angular velocities. Solving (1) for $i, k$, in terms of $i^{\prime}, k^{\prime}$, substituting in (2) and equating coefficients of like vectors we get

$$
\begin{aligned}
& v^{\prime}(t) \tau^{\prime}(t)=v(t) \tau(t) \cos \theta+v(t) \kappa(t) \sin \theta, \\
& v^{\prime}(t) \kappa^{\prime}(t)=-v(t) \tau(t) \sin \theta+v(t) \kappa(t) \cos \theta .
\end{aligned}
$$

Nothing is lost in generality if we identify the arc length along the curve $C$ with time, thus letting $v(t)=1$.

Having chosen $\theta$, the successive positions of $I^{\prime}$ and consequently also of $i^{\prime}$ are determined as functions of $t$. The curve $C^{\prime}$ itself is not specified till we give $v^{\prime}=d s / d t$, whereupon we obtain $C^{\prime}$ by vectorial integration of $v^{\prime}(t) i^{\prime}(t)$ (one must also give the starting point). All the curves that we obtain from a fixed $\theta$ by varying $v^{\prime}$ can be made to correspond to each other, so that at corresponding points (given by the same values of $t$ ), the indicatrix trihedra coincide, and are thus to each other in relation of Combescure.

We shall restrict ourselves for the present to the case where $v^{\prime}=1$, but will let $\theta$ take on any value between 0 and $2 \pi$; the curves $C^{\prime}$, which we are seeking, will have any two of their points the same arc length apart as the points of $C$ to which they correspond. We may now put $s^{\prime}=s=t$ where $s^{\prime}$ is the arc length along $C$; (3) reduces to

$$
\begin{aligned}
& \tau^{\prime}(t)=\tau(t) \cos \theta+\kappa(t) \sin \theta, \\
& \kappa^{\prime}(t)=-\tau(t) \sin \theta+\kappa(t) \cos \theta .
\end{aligned}
$$

If we start all the curves $C^{\prime}$ with the point $s^{\prime}=0$ at a common starting point $Q$, there will be obtained a oneparameter family of curves $F$, one curve corresponding to every value of the parameter $\theta$. The given curve $C$ corresponds to $\theta=0$. All other curves that have an indicatrix trihedron $I^{\prime}$ fixed relative to $I$ may be obtained from some one curve of $F$ by a transformation of Combescure. ( $\left(3^{\prime}\right)$ shows 
that the curvatures and torsions of the curves of $F$ are certain linear combinations of those of the initial curve. The family $F$ has some interesting properties which we now proceed to investigate.

Denot: by $\boldsymbol{P}(\theta, s)$ the vector drawn from the point $Q$ to any point $P^{\prime}$ on the curves $F$; similarly replace $i^{\prime}(t), j^{\prime}(t)$, $\boldsymbol{k}^{\prime}(t)$ by $\boldsymbol{i}(\theta, s), \boldsymbol{j}(\theta, s), \boldsymbol{k}(\theta, s)$. Between $\boldsymbol{P}$ and $\boldsymbol{i}$ we have the relation

$$
\frac{\partial \boldsymbol{P}(\theta, s)}{\partial s}=\boldsymbol{i}(\theta, s),
$$

whence by integration, since $\boldsymbol{P}(\theta, 0)=0$,

$$
\boldsymbol{P}(\theta, s)=\int_{0}^{s} i(\theta, s) d s .
$$

Using (1) we obtain

$$
\boldsymbol{P}(\theta, s)=\cos \theta \int_{0}^{s} \boldsymbol{i}(0, s) d s+\sin \theta \int_{0}^{s} \boldsymbol{k}(0, s) d s .
$$

Since $\boldsymbol{k}(\theta, s)=\boldsymbol{i}\left(\theta+\frac{\pi}{2}, s\right)$, (5) becomes

$$
\boldsymbol{P}(\theta, s)=\cos \theta \int_{0}^{s} i(0, s) d s+\sin \theta \int_{0}^{s} i\left(\frac{\pi}{2}, s\right) d s,
$$

and, using $\left(4^{\prime}\right)$, we find

$$
\boldsymbol{P}(\theta, s)=\cos \theta \boldsymbol{P}(0, s)+\sin \theta \boldsymbol{P}\left(\frac{\pi}{2}, s\right) .
$$

If we hold $s$ constant and let $\theta$ run between 0 and $2 \pi$, the end point of $\boldsymbol{P}(\theta, s)$ will describe the locus of the corresponding points on the curves $F$, which are at an arc length $s$ from $Q$. From (6) we conclude that these corresponding points always lie on ellipses $E$. The latter have their centers at $Q$, and the vectors $\boldsymbol{P}(0, s), \boldsymbol{P}(\pi / 2, s)$ are conjugate semi-axes. Obviously, any two curves whose values of $\theta$ differ by $\pi / 2$ will yield conjugate axes of the ellipses. We might call such curves conjugates of each other. The tangents to two conjugate curves are perpendicular to each other.

When $s$ is small the ellipses approximate a circle. For, from (5), $\boldsymbol{P}(\theta, s)=[\cos \theta \boldsymbol{i}(0,0)+\sin \theta \boldsymbol{k}(0,0)] s$ approximately for small $s$. The plane of this circle is perpendicular to the common principal normal of $F$ at $Q$.

In the plane $\pi(0)$ through $i(0,0), \boldsymbol{k}(0,0)$, let us set up a 
system of rectangular Cartesian axes $(x, y)$ with origin at the vertex of $I$, the end-points of the two unit vectors mentioned having coordinates $(1,0),(0,1)$, respectively. The unit circle described by $\boldsymbol{i}(\theta, 0)$ now has for its parametric equations

$$
x=\cos \theta, \quad y=\sin \theta .
$$

Similarly, in the plane of the ellipse $E$, described by $\boldsymbol{P}(\theta, s)$ when $s$ is held fixed, let us set up a system of oblique Cartesian coordinates $(\xi, \eta)$ the origin being at $Q$, while the axes and scales on them are chosen so that the coordinates of $\boldsymbol{P}(0, s)$, $\boldsymbol{P}(\pi / 2, s)$ are $(1,0)$ and $(0,1)$, respectively. Any vector $a \boldsymbol{P}(0, s)+b \boldsymbol{P}(\pi / 2, s)$ will then have for the coordinates of its end-points $\xi=a, \eta=b$. Hence, using (6) we get for the parametric equations of the ellipse,

$$
\xi=\cos \theta, \quad \eta=\sin \theta .
$$

Now consider any affine projective transformation carrying the plane $(x, y)$ into $(\xi, \eta)$ so that for corresponding points $x=\xi$ and $y=\eta$. Such an affine projective transformation will send the circle (7) into the ellipse (8), corresponding points being given by the same value of $\theta$. Therefore, corresponding points on two ellipses are projectively related. If we join such points by drawing corresponding secants for the curves, that is, secants between two corresponding points, we obtain a ruled surface of (not higher than) the fourth degree. By passing to the limit we get a similar result for the tangents.

The result obtained that points of $F$ lie on ellipses $E$ is only a special case of a more general theorem. If we call $M$ the motion described by the moving trihedron of $F$ as $\theta$ is varied but $s$ is held fixed, and denote by $R$ any point fixed to that trihedron, then the path of $R$ as a result of the motion $M$ will be an ellipse. For

$$
\begin{aligned}
\overrightarrow{Q R} & =\overrightarrow{Q P}+\overrightarrow{P R} \\
& =\boldsymbol{P}(\theta, s)+c_{1} i(\theta, s)+c_{2} j(\theta, s)+c_{3} k(\theta, s),
\end{aligned}
$$

where $c_{1}, c_{2}, c_{3}$ are constants. Using (1) and (6) we obtain

$$
\begin{aligned}
\overrightarrow{Q R}= & \cos \theta\left[\boldsymbol{P}(0, s)+c_{1} i(0, s)+c_{3} k(0, s)\right] \\
& +\sin \theta\left[P\left(\frac{\pi}{2}, s\right)+c_{1} i\left(\frac{\pi}{2}, s\right)+c_{3} k\left(\frac{\pi}{2}, s\right)\right]+c_{2} j(0, s) ;
\end{aligned}
$$


$s$ being held fixed $=s_{0}$, this is the equation of an ellipse having its center at $c_{2} j\left(0, s_{0}\right)$, that is, on the line through $Q$ having the direction of the principal normals at the points $\boldsymbol{P}\left(\theta, s_{0}\right)$.

The movements $M$ in which all points describe ellipses are of the type described by Darboux in one of his notes to Koenigs' Kinematics. ${ }^{*}$ It is there proved that this is the only possible movement in which the trajectories of all points are plane curves, except for the case where the trajectories lie in a family of parallel planes. A line generally describes a surface of the fourth degree. Such a motion may be generated by proper rolling and slipping of two circular cylinders whose radii are in the ratio $1: 2$. The axes of these cylinders will in the present instance be parallel to the principal normals.

Since the relation $\left(3^{\prime}\right)$ between $\kappa, \tau$ and $\kappa^{\prime}, \tau^{\prime}$ is the same as between the coordinates of the same point in a plane referred to two sets of rectangular axes with a common origin and forming an angle $\theta$ with each other, it follows that if $\kappa, \tau$ satisfy an algebraic relation, $\kappa^{\prime}, \tau^{\prime}$ will satisfy a relation of the same degree. Thus, if $\kappa, \tau$ satisfy a linear relation so that $C$ is a Bertrand curve, all the curves of $F$ will be Bertrand curves. There will then exist two values of $\theta$ for which $\kappa^{\prime}=$ const., and two other values differing from the former by $\pi / 2$ for which $\tau^{\prime}=$ const. The latter curves are the conjugates of the former.

The expression $\left[\kappa^{2}+\tau^{2}\right]^{1 / 2}$ gives the rate at which the principal normal or the rectifying plane turns, and is sometimes spoken of as the "normal" curvature. From (3) it follows that for all the curves of $F$, the normal curvature is the same. In particular, if it is constant for $C$, it is equal to the same constant for any curve of $F$.

$F$ was obtained by putting $v^{\prime}=d s^{\prime} / d s$ equal to unity, that is, by letting corresponding points on $C$ and $C^{\prime}$ be equal arc lengths apart. Other interesting families of curves may, no doubt, be obtained by choosing other proper expressions for $v^{\prime}$. Thus, if we let $v^{\prime}$ be a function of $\theta$ only, $v^{\prime}=v(\theta)$, we can go through a treatment similar to that for the $F$ family. Since

* G. Koenigs, Leçons de Cinématique, Note IV, §§ 2, 3, p. 252. 
we may no longer use the same symbol $s$ to denote arc length along any curve, we shall think of corresponding points along different curves as given by the same value of $t$. We now have (in place of (4))

$$
\frac{\partial \boldsymbol{P}(\theta, t)}{\partial t}=\frac{\partial \boldsymbol{P}(\theta, t)}{\partial s^{\prime}} \frac{d s^{\prime}}{d t}=v(\theta) \cdot \frac{\partial \boldsymbol{P}}{\partial s^{\prime}}=v(\theta) \boldsymbol{i}(\theta, t) ;
$$

and, instead of (6),

$$
\boldsymbol{P}(\theta, t)=\cos \theta v(\theta) \boldsymbol{P}(0, t)+\sin \theta v(\theta) \boldsymbol{P}\left(\frac{\pi}{2}, t\right) .
$$

This is the locus of corresponding points on the curves now. It is still a plane curve and may be regarded as an affine projective image of the curve whose equation in polar coordinates is $\rho=v(\theta)$, this curve taking the place of the unit circle of which the ellipses of family $F$ were projective images.

In case $\rho=v(\theta)$ is an algebraic curve, it can be shown that the movements $M$, described by the moving trihedra of the curves as $\theta$ varies while $t$ is fixed, are still algebraic, that is, the path of any point during this motion is an algebraic curve; its degree is considerably higher than that of $\rho=v(\theta)$. Thus if we take $\rho=\sec \theta$, the corresponding points of the curves always lie on straight lines. During the motion $M$ the general point can be shown to describe a curve of the fourth degree which is an affine projective image of

$$
z=\tan \theta, \quad x=\cos \theta, \quad y=\sin \theta .
$$

Now let us return to the case where $C$ is a cylindrical helix, the motion $[I, S]$ reducing to a rotation of $I$ about a fixed axis $l$ (whose direction gives the direction of the rulings of the cylinder). The positions $I^{\prime}$ could take up relative to $I$ are now of two parameters instead of one. For, if the position of $i$ be arbitrarily assigned, since the rotation vector lying on $l$ must always be in the plane $i^{\prime}, k^{\prime}$, the position of $I^{\prime}$ is completely specified (except for the case when $i$ coincides with $l$ ). $I^{\prime}(t)$ is now determined in time, and it remains only to choose $v^{\prime}(t)$ and the initial point in order to specify $C^{\prime}$. Whichever $I^{\prime}(t)$ we pick out, it rotates about $l, i$ forming a constant angle with it. Hence all the curves $C^{\prime}$ are cylindrical helices, the 
elements of whose cylinders are parallel to those of $C$. The curves obtained by putting $v^{\prime}=1$, and prescribing a common starting point $Q$ form a two-parameter family of curves $G$, such that any other curve whose indicatrix $I^{\prime}$ is fixed relative to $I$ is in a relation of Combescure to some curve of $G$.

If we restrict $i$ to lie in a plane through $l$, we pick out a one-parameter group of positions of $I$, which is of the type previously discussed for a general curve $C$. We may now construct the family $F$ by picking out a position of $i$ to measure $\theta$ from. For two values of $\theta$ (differing by $\pi$ ) the unit tangent will coincide with $l$, yielding for the curve $C$ a straight line $C_{s}$. The two curves $C_{p}$ that are conjugate to $C_{s}$ will have their tangents perpendicular to the line $C_{s}$, and consequently lie in a plane perpendicular to $C_{s}$. The vectors joining $Q$ to corresponding points on $C_{s}, C_{p}$ will form conjugate axes of the ellipse $E$, and being perpendicular to each other will now be the major and minor axes of $E$. The points of $F$ can thus be obtained by having an ellipse move so that its major axis increases at a uniform rate along a straight line while the minor axis describes a plane curve with the same velocity.

If we now rotate about $l$ the plane in which we restricted $i$ to lie, all possible positions of $I^{\prime}$ (relative to $I$ ) are obtained. The infinitesimal vectors $i d s$ experience a fixed rotation about $l$, and the same is true of the sum vector $\mathcal{S}_{0}{ }^{8} i d s$. Hence, all the other curves of $G$ may be obtained from the one-parameter family just considered by rotating the latter about $l$.

We see, therefore, that if we hold $s$ constant, the locus of a point fixed relative to $I^{\prime}$ is a surface of the fourth degree obtained by revolving an ellipse about the line $l$. In particular the locus of the vertex of $I^{\prime}$ which describes the curves $C$ themselves reduces to an ellipsoid of revolution since the ellipse has its major axis on $l$. The torsions and curvatures of $C^{\prime}$ are again linear combinations of those of $C$, the relation being given by $\left(3^{\prime}\right)$. Hence, if $C$ is a circular helix, so that $\tau, \kappa$ are constant, $\tau^{\prime}, \kappa^{\prime}$ will also be constant, and all the curves of $G$ will be circular helices.

CoRNell UNIVERSITY 